\title{
Mumps Outbreak at a University and Recommendation for a Third Dose of Measles-Mumps-Rubella Vaccine — Illinois, 2015-2016
}

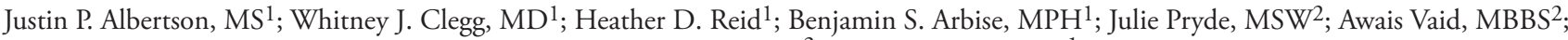 \\ Rachella Thompson-Brown ${ }^{2}$; Fredrick Echols, MD ${ }^{1}$
}

Mumps is an acute viral disease characterized by fever and swelling of the parotid or other salivary glands. On May 1, 2015, the Illinois Department of Public Health (IDPH) confirmed a mumps outbreak at the University of Illinois at Urbana-Champaign. IDPH and the Champaign-Urbana Public Health District (C-UPHD) conducted an investigation and identified 317 cases of mumps during April 2015-May 2016. Because of sustained transmission in a population with high 2-dose coverage with measles-mumps-rubella (MMR) vaccine, a third MMR dose was recommended by IDPH, C-UPHD, and the university's McKinley Health Center. No formal recommendation for or against the use of a third MMR dose has been issued by the Advisory Committee on Immunization Practices (ACIP) (1). However, CDC has provided guidelines for use of a third dose as a control measure during mumps outbreaks in settings in which persons are in close contact with one another, where transmission is sustained despite high 2-dose MMR coverage, and when traditional control measures fail to slow transmission (2).

On April 15, 2015, the university health center reported to C-UPHD a male aged 21 years with fever and parotitis beginning April 9. Mumps was suspected; however, confirmatory testing was not performed. During the following 2 weeks, five additional suspected cases of mumps were identified. Each patient received a diagnosis of parotitis without laboratory confirmation of mumps. All patients with suspected mumps had documentation of receipt of 2 doses of MMR vaccine. On May 1, 2015, a seventh suspected mumps case was confirmed by a positive real-time reverse transcription-polymerase chain reaction (rRT-PCR) test of a buccal swab conducted at the IDPH state laboratory. The six previous suspected cases were epidemiologically linked to the same academic program as the confirmed case, which enabled IDPH to establish the existence of a mumps outbreak at the university.

Confirmed, probable, and suspected cases were identified using the standard case definition for mumps (3). Patients were considered to be infectious from 2 days before until 5 days after the onset of parotitis. The exposure period was defined as 12-25 days before the onset of parotitis. Outbreak control measures recommended to the university health center by C-UPHD included standard and droplet precautions for patients in health care facilities and isolation of ill patients. Ill students were directed to return home or were provided alternative housing during their infectious period. Investigators identified contacts of mumps patients to verify receipt of 2 doses of MMR vaccine and recommended vaccination of susceptible close contacts if they were not fully vaccinated.* Susceptible close contacts who had a contraindication to vaccination or who refused vaccination were excluded from public settings for 14 days (from days 12-25 following exposure to a person with probable or confirmed mumps). University vaccination records were reviewed, and 2-dose MMR vaccination coverage was estimated at $>97 \%$ among all students. On May 26, 2015, IDPH posted a notification on CDC's Epidemic Information Exchange (Epi-X) and issued a memorandum to health departments to request information on cases among persons who returned home from the university during the summer semester.

Despite high 2-dose MMR coverage and a reduced student population on campus, cases continued to occur during the summer semester (Figure). By July 31, a total of 70 cases had been reported. On August 4, IDPH, C-UPHD, and the university health center issued a recommendation for all students and staff members born during or after $1957^{\dagger}$ to receive an additional dose of MMR vaccine (2). Notifications were sent to students and their families, and an Epi-X notification was posted to inform state health agencies of the recommendation. An estimated 50,000 students and staff members were targeted for this intervention.

A total of 8,200 doses of MMR vaccine were administered at five vaccination clinics held on the university campus during August 6-27. An unknown number of additional vaccine doses were administered to students and staff members living off-campus during the summer, who were encouraged to received vaccine from a health care provider or pharmacy before returning to school. C-UPHD and the university health center administered an additional 3,300 doses throughout the

\footnotetext{
* Susceptible persons include all persons without evidence of immunity to mumps. Evidence of immunity includes 1) laboratory evidence of immunity to mumps; 2) documentation of receipt of 2 doses of MMR vaccine for school-aged children and adults at high risk (e.g., health care personnel, international travelers, or students at postsecondary educational institutions); 3) documentation of at least 1 dose of MMR vaccine for preschool-aged children and adults not at high risk; 4) birth before 1957; or 5) documentation of physician-diagnosed mumps.

$\dagger$ Because of widespread transmission of the mumps virus before the mumps vaccine was recommended for routine use, persons born before 1957 are likely to have been infected naturally and are presumed to be immune.
} 
FIGURE. Number of confirmed and probable cases of mumps $(\mathrm{N}=317)$ on the University of Illinois at Urbana-Champaign campus, by month of onset - Illinois, April 2015-May 2016

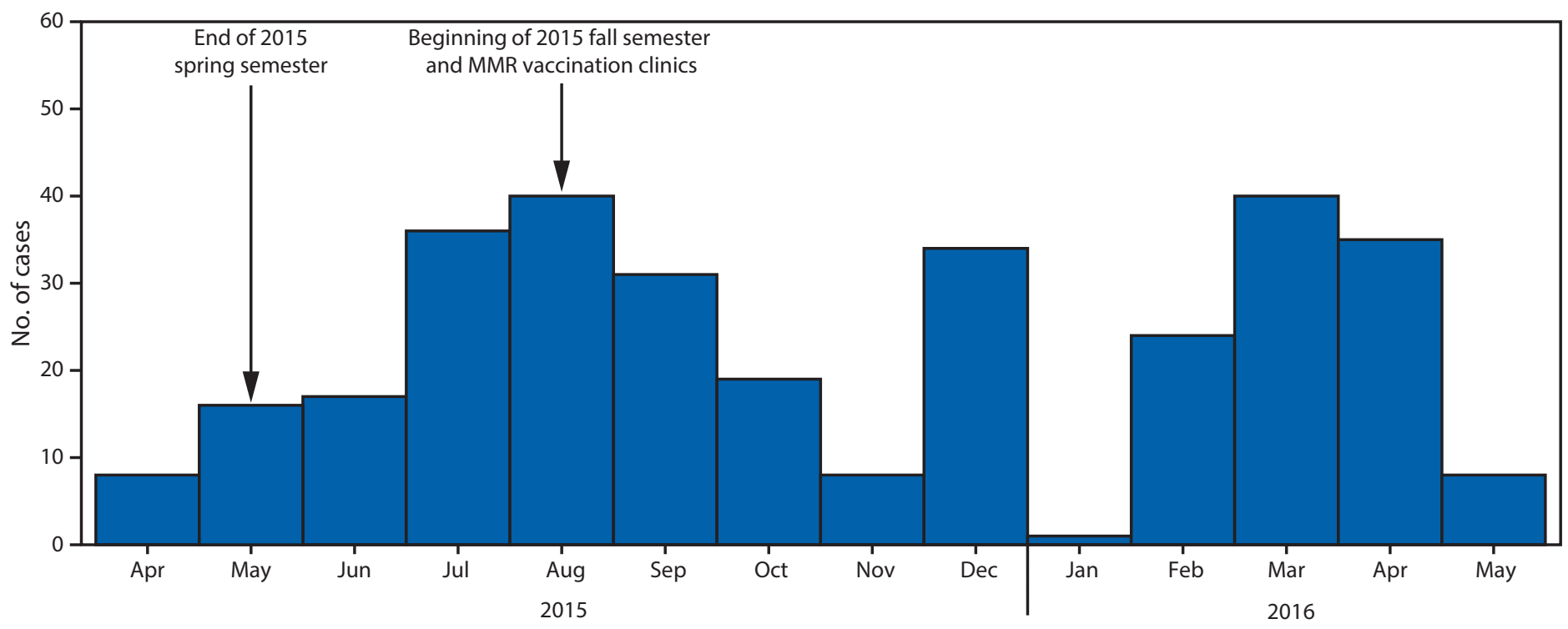

Month/Year

Abbreviation: $\mathrm{MMR}=$ measles, mumps, rubella.

fall and spring semesters. Persons vaccinated were monitored for 15 minutes after receiving the vaccine and were given a vaccine information statement. No serious vaccine-related adverse events were reported.

Investigators identified 317 probable and confirmed mumps cases with onset during April 9, 2015-May 27, 2016. One hundred (32\%) cases were laboratory confirmed by rRT-PCR, and $217(68 \%)$ were classified as probable. Cases occurred in persons who ranged in age from $16-55$ years, with a median age of 20 years. Twenty-two (7\%) patients were evaluated at the emergency department, and three $(1 \%)$ were hospitalized (one to treat meningitis, one to rule out meningitis, and one for parotitis pain management). Two (1\%) patients experienced orchitis, a recognized complication of mumps (2). No deaths were reported. Specimens from four cases were genotyped at the CDC Measles, Mumps, Rubella, and Herpesvirus Laboratory Branch; all were mumps genotype G. All cases were epidemiologically linked to the university; 278 (88\%) cases occurred in students, three $(1 \%)$ in staff members, and $36(11 \%)$ in persons not affiliated with the university, but who had contact with university students or the campus. Several sub-clusters occurred within the larger outbreak in certain academic programs, athletic facilities, and community workplaces.

Among the 317 cases identified, at the time of parotitis onset, $50(16 \%)$ mumps patients had received 3 doses of MMR vaccine, 232 (73\%) had received 2 doses, 12 (4\%) had received 1 dose, seven (2\%) were unvaccinated, and 16 (5\%) had unknown vaccination status. Forty-five $(90 \%)$ of the
50 patients with a third dose received it during this outbreak, and five $(10 \%)$ received it in prior years for reasons unrelated to this outbreak. Some of the 45 persons who received a third dose during this outbreak might have been exposed before vaccine-induced immunity was boosted. Eleven (24\%) of the 45 patients had parotitis onset on the same day or within 2 weeks after receiving the third dose, six (13\%) within $2-4$ weeks, and $27(60 \%)>4$ weeks after. One (2\%) patient received a third dose 3 days after parotitis onset.

\section{Discussion}

As in many previously described mumps outbreaks, this outbreak was characterized by sustained transmission, despite high 2-dose MMR vaccination coverage (4-7). Two doses of MMR vaccine are currently routinely recommended for the prevention of mumps; the first for children at 12-15 months of age and the second for children at 4-6 years of age (2). The median vaccine effectiveness against mumps has been estimated at $78 \%$ for 1 dose and $88 \%$ for 2 doses (2). However, 2-dose vaccine failure and possible waning of vaccine-induced immunity have been described in recent outbreaks, particularly in high-density, close-contact settings (4-8). Because outbreaks occur despite high 2-dose coverage, a third dose has been provided as a control measure to targeted populations during previous outbreaks $(4,5,7)$.

No formal recommendation for a third MMR dose exists, but $\mathrm{CDC}$ has provided guidelines for public health agencies considering its use as a control measure during mumps outbreaks (2). 
Factors that might trigger a recommendation include outbreaks among populations with 2-dose MMR vaccination coverage of $>90 \%$, intense exposure settings such as universities, evidence of sustained transmission for $>2$ weeks, and high attack rates ( $>5$ cases per 1,000 population). Evidence of sustained disease transmission despite high 2-dose vaccination coverage among university students supported the decision to recommend a third MMR dose during this outbreak. The fourth criterion of high attack rates was not considered; because transmission occurred during spring, summer, and fall semesters when student enrollment varied widely, it was not possible to calculate an accurate attack rate because of frequent, large changes in the denominator.

In addition to meeting criteria in the CDC guidelines, two important aspects of this outbreak supported the recommendation for a third dose of MMR vaccine. First, this outbreak did not follow typical seasonal trends for mumps in Illinois, where incidence normally peaks during late winter and spring (Illinois Department of Public Health, unpublished data, 2016); evidence of sustained transmission extending into the summer months was concerning. During 2005-2014, the median number of mumps cases each year during June and July in Illinois was six statewide; in this outbreak, 53 cases occurred during these 2 months. This deviation from the 10-year median and continued transmission during the summer months was unexpected because of the reduction of high-density, closecontact settings on campus. Student enrollment declined from 41,497 in the 2015 spring semester to 11,684 in the summer, a reduction of $72 \%$.

The second aspect that supported the recommendation was the anticipation of a large number of students returning for the 2015 fall semester, which would increase the population density on campus and provide opportunities for exposure. Many students would be returning to high-density congregate settings such as university housing, and large social events often occur early during the semester. An unknown number of susceptible persons would also be added to the population; although documentation of mumps vaccination (or other evidence of mumps immunity) is required by the university, the requirement is not enforced until students attempt to register for the subsequent semester.

The effectiveness of a third dose of MMR vaccine has not been established, but rationale exists for its use in outbreak settings. There is some evidence that a third dose induces an immune response, and in two outbreaks, attack rates declined after a third dose intervention $(4,5,9,10)$. However, the decline in attack rate was not statistically significant in one outbreak; in both outbreaks, the intervention was given after the outbreaks

\section{Summary \\ What is already known on this topic?}

Mumps outbreaks can occur in close-contact settings like universities, despite high 2-dose MMR vaccination coverage. A third dose of MMR vaccine has been used in previous mumps outbreaks, but its effectiveness is not established.

What is added by this report?

A large outbreak of mumps occurred at the University of Illinois at Urbana-Champaign during April 2015-May 2016; 89\% of patients with mumps had received at least 2 doses of measles-mumpsrubella (MMR) vaccine, and a third dose was recommended as a control measure. The rationale for the recommendation of a third MMR dose included a consideration of seasonal trends and characteristics of the at-risk population. These were weighed against potential drawbacks, which included the potential for vaccine-related side effects, associated costs, and the lack of evidence of the effectiveness of a third MMR dose.

What are the implications for public health practice?

Both CDC guidelines and factors unique to the outbreak should be carefully considered by public health agencies before issuing a similar recommendation. Additional studies are needed to determine the effectiveness of a third MMR dose as a mumps outbreak control measure in certain populations.

had peaked, and other outbreaks occurring among similar populations showed declining attack rates without administration of a third dose of MMR vaccine (8). In addition, recommending an intervention that has limited evidence of effectiveness might result in unnecessary costs and introduce the potential for vaccine-related adverse events. Currently there is no formal recommendation for a third dose of MMR vaccine during mumps outbreaks; the decision to implement this intervention needs to be carefully considered. In light of the recent increased incidence of mumps, CDC is gathering additional data to assess use of a third dose of vaccine to inform decision-making during outbreak responses and potential changes in the recommendations.

Although evidence of its effectiveness is needed, a third dose of MMR vaccine may be considered as a control measure during mumps outbreaks occurring in settings in which persons are in close contact with one another, when transmission is sustained despite high 2-dose MMR coverage, and when traditional control measures fail to slow transmission. The final case in this outbreak occurred in May 2016, and the outbreak was declared over in July. Although transmission continued until May 2016, there was a decline in cases in the months immediately following August 2015, when the recommendation was made. Further evaluation is needed to determine if the reduction was a result of the recommendation for a third MMR dose. 


\section{Acknowledgments}

McKinley Health Center staff members, University of Illinois at Urbana-Champaign; Chicago Department of Public Health, Skokie Health Department, health departments of Cook, DeKalb, DuPage, Kane, Lake, Logan, and Will counties, Illinois; Connie Austin, Craig Conover, Carol Finley, Kathleen Kelly-Shannon, Lori Saathoff-Huber, Illinois Department of Public Health; Division of Viral Diseases, National Center for Immunization and Respiratory Diseases, CDC.

${ }^{1}$ Illinois Department of Public Health; ${ }^{2}$ Champaign-Urbana Public Health District.

Corresponding author: Heather D Reid, Heather.Reid@Illinois.gov, 217-782-2016.

\section{References}

1. McLean HQ, Fiebelkorn AP, Temte JL, Wallace GS. Prevention of measles, rubella, congenital rubella syndrome, and mumps, 2013: summary recommendations of the Advisory Committee on Immunizations Practices (ACIP). MMWR Recomm Rep 2013;62(No. RR-04).

2. Fiebelkorn AP, Barskey A, Hickman C, Bellini W. Mumps [Chapter 9]. In: VPD surveillance manual. 5th ed. Atlanta, GA: US Department of Health and Human Services, CDC; 2012. http://www.cdc.gov/vaccines/ pubs/surv-manual/chpt09-mumps.pdf

3. Council of State and Territorial Epidemiologists. Public health reporting and national notification for mumps. Position statement: 11-ID-18. Atlanta, GA: Council of State and Territorial Epidemiologists; 2011. http://c.ymcdn. com/sites/www.cste.org/resource/resmgr/PS/11-ID-18.pdf
4. Ogbuanu IU, Kutty PK, Hudson JM, et al. Impact of a third dose of measles-mumps-rubella vaccine on a mumps outbreak. Pediatrics 2012;130:e1567-74. http://dx.doi.org/10.1542/peds.2012-0177

5. Nelson GE, Aguon A, Valencia E, et al. Epidemiology of a mumps outbreak in a highly vaccinated island population and use of a third dose of measles-mumps-rubella vaccine for outbreak control-Guam 2009 to 2010. Pediatr Infect Dis J 2013;32:374-80. http://dx.doi. org/10.1097/INF.0b013e318279f593

6. Cortese MM, Jordan HT, Curns AT, et al. Mumps vaccine performance among university students during a mumps outbreak. Clin Infect Dis 2008;46:1172-80. http://dx.doi.org/10.1086/529141

7. Zipprich J, Murray EL, Winter K, et al. Mumps outbreak on a university campus-California, 2011. MMWR Morb Mortal Wkly Rep 2012;61:986-9.

8. Dayan GH, Quinlisk MP, Parker AA, et al. Recent resurgence of mumps in the United States. N Engl J Med 2008;358:1580-9. http://dx.doi. org/10.1056/NEJMoa0706589

9. Date AA, Kyaw MH, Rue AM, et al. Long-term persistence of mumps antibody after receipt of 2 measles-mumps-rubella (MMR) vaccinations and antibody response after a third MMR vaccination among a university population. J Infect Dis 2008;197:1662-8. http://dx.doi. org/10.1086/588197

10. Fiebelkorn AP, Coleman LA, Belongia EA, et al. Mumps antibody response in young adults after a third dose of measles-mumps-rubella vaccine. Open Forum Infect Dis 2014;1:ofu094. http://dx.doi. org/10.1093/ofid/ofu094 